\title{
An unusual insertion of an accessory band of the semitendinosus tendon: case report and review of the literature
}

\author{
Ł. Olewnik¹, M. Podgórski², M. Polguj ${ }^{3}$ \\ ${ }^{1}$ Department of Normal and Clinical Anatomy, Interfaculty Chair of Anatomy and Histology, Medical University of Lodz, Poland \\ 2Department of Diagnostic Imaging, Polish Mother's Memorial Hospital Research Institute, Lodz, Poland \\ ${ }^{3}$ Department of Angiology, Interfaculty Chair of Anatomy and Histology, Medical University of Lodz, Poland
}

[Received: 24 June 2019; Accepted: 2 July 2019]

The Pes Anserinus is characterised by high morphological variability. It consists of three tendons: the sartorius, gracilis and semitendinosus. The semitendinosus and gracilis tendons are routinely harvested for reconstruction anterior cruciate ligament, and the presence of accessory bands within them can handicap the harvesting process. This report presents a case of a rare insertion of accessory band of the semitendinosus tendon (to the fascia of the soleus muscle and tibia). The current classification should be extended to accommodate such "rare cases" to facilitate more successful hamstring grafts. (Folia Morphol 2020; 79, 3: 645-648)

Key words: hamstring graft, harvest, pes anserinus, semitendinosus tendon, gracilis tendon

\section{INTRODUCTION}

The semitendinosus muscle is one of the hamstring muscles located in the postero-medial side of the thigh. It originates from the lower and medial impression on the superior part of the tuberosity of the ischium, through a tendon common to the ischium itself and the long head of the biceps femoris [12, 25]. The belly of the semitendinosus becomes a long tendon, which is involved in the formation of the Pes Anserinus (PA) [18, 25].

The PA is anatomically defined as the conjoined tendons of three muscles: the sartorius tendon (ST), gracilis tendon (GT) and the semitendinosus tendon (STT). At the distal part of the PA, at the tibial attachment, the ST, GT and STT fuse with the fascia of the leg, forming the Anserinus plate [18]. The PA consists of a superficial layer formed by the ST, and a deep layer formed by the GT and STT [18].
A strong autograft can be created with hamstring tendons. The STT and the GT are commonly used as autograft material for the reconstruction of the anterior cruciate ligament $(\mathrm{ACL})[3,10,18$, $23,29]$ or patellar tendon after rupture $[14,18$, 26]. Nonetheless, complications associated with PA tendon transplantation have been reported, and a classification has been proposed to clarify the morphological variations occurring as additional bands [18].

On rare occasions, unexpected types of accessory band can be encountered in the STT, as described in the present report. We therefore propose to extend the existing classification of PA by including 'rare cases'. It would allow for such further variations when harvesting autograft material and to improve the planning of orthopaedic surgery in this area. 


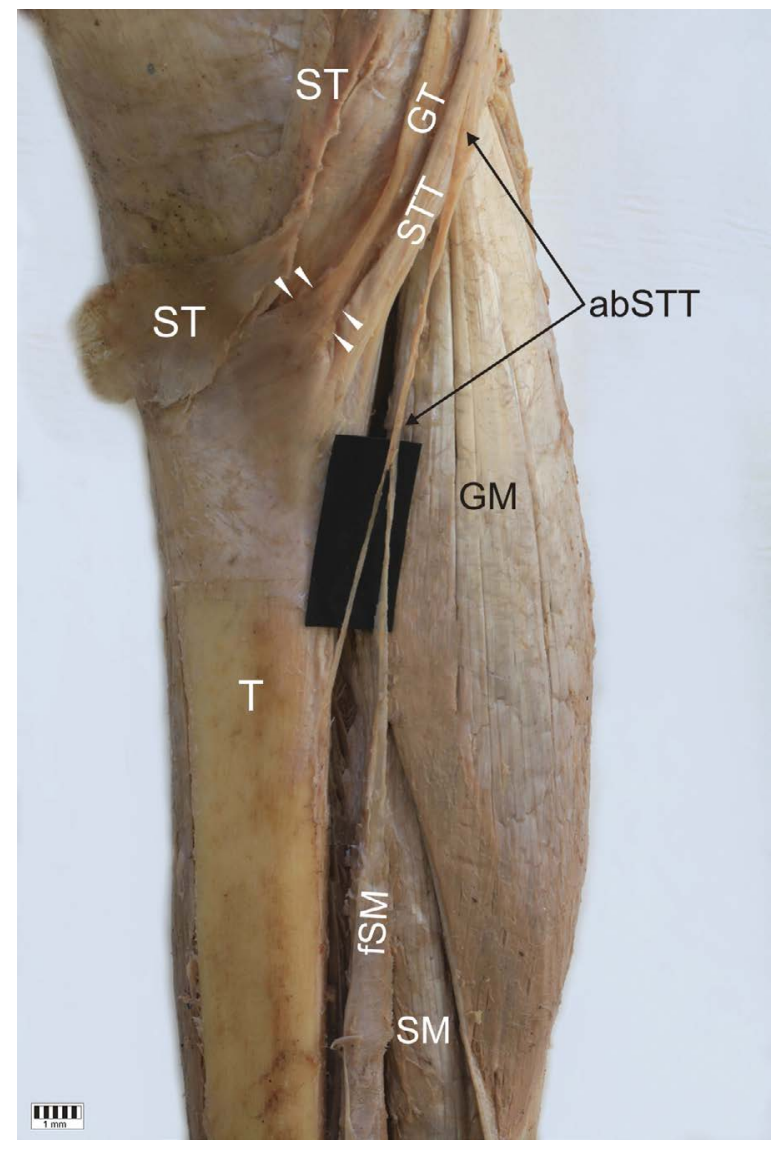

Figure 1. Rare anatomical type of the semitendinosus tendon (STT); ST — sartorius tendon; GT — gracilis tendon; abSTT — accessory band of the semitendinosus tendon; GM — gastrocnemius muscle; $\mathrm{T}$ - tibia; $\mathrm{fSM}$ - fascia of the soleus muscle.

\section{CASE REPORT}

A female cadaver, 69 years old at death, was subjected to routine anatomical dissection for research and teaching purposes at the Department of Normal and Clinical Anatomy, Medical University of Lodz. The dissection of the thigh, knee and crural region was performed using standard techniques according to a strictly specified protocol [16-20]. During the dissection of the right medial thigh and crural region, an unusual accessory band and insertion of the semitendinosus muscle was found. Morphometric measurements were taken based on digital photographic images processed through MultiScanBase 18.03 (Computer Scanning System II, Warsaw, Poland).

The accessory band was localised $80.79 \mathrm{~mm}$ from the main PA insertion. The length of the additional band to the split was $74.02 \mathrm{~mm}$. The shorter band (length $49.91 \mathrm{~mm}$ ) inserted into the tibia, while the longer band $(84.77 \mathrm{~mm})$ inserted into the fascia of the soleus muscle (Figs. 1, 2).

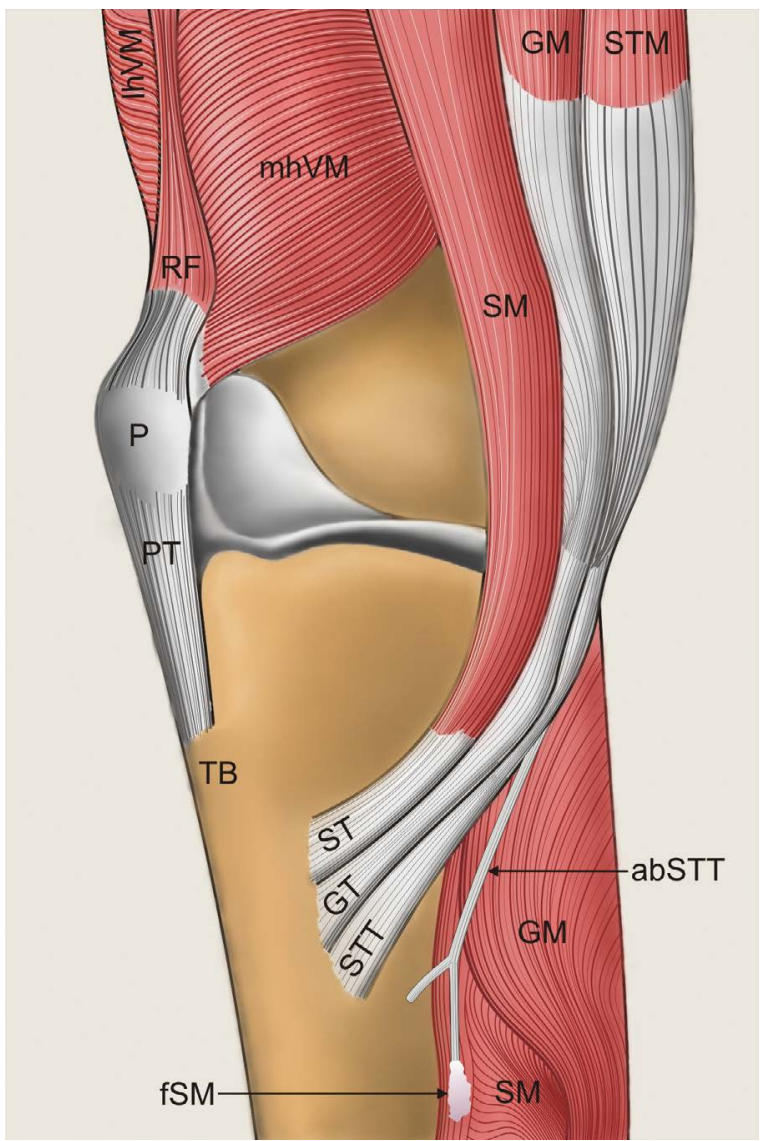

Figure 2. Schematic drawing of the Pes Anserinus and the accessory band of the semitendinosus tendon (STT); IhVL — lateral head of the vastus lateralis; mhVM - medial head of the vastus medialis; RF — rectus femoris muscle; $\mathrm{P}$ — patella; $\mathrm{PT}$ - patellar tendon; TB — tibial tuberosity; SM — sartorius muscle; ST — semitendinosus tendon; GM — gracilis muscle; GT — gracilis tendon; STM — semitendinosus muscle; GM — gastrocnemius muscle; abSTT - accessory band of the semitendinosus tendon; SM - soleus muscle; fSM — fascia of the soleus muscle.

The origin and course of the semitendinosus muscle were normal, as was the insertion into the tibia. The length of the muscle belly was $281.98 \mathrm{~mm}$, while the length of the tendon was $154.43 \mathrm{~mm}$. The STT displayed a band-shaped insertion, with its innervation taking place from a muscular branch of the tibial nerve. The ST displayed normal origin and course and was inserted into the tibia. The GT presented a fan-shaped insertion. No other anatomical variations were identified. The type of insertion was determined using types as described previously [18].

\section{DISCUSSION}

Injuries of the $\mathrm{ACL}$ account for anywhere between $25 \%$ and $50 \%$ of all ligamentous knee injuries, and $A C L$ reconstruction is one of the most common orthopaedic techniques performed worldwide [6, 8, 21, 24]. 
While the fibular and tibial collateral ligaments exhibit strong healing potential and generally respond well to conservative treatment, the $\mathrm{ACL}$ has poor intrinsic healing ability due to the fact that it is enveloped by synovial fluid and lacks significant vascularisation $[9,13,17]$. Therefore, reconstruction seems to be the best current solution for ACL tears, with autograft reconstruction using the patellar tendon or quadrupled GT/STT being most popular procedure used for ACL surgery $[9,13,14,18,21,22,26]$.

While both patellar graft and hamstring tendon graft have their advantages and disadvantages, hamstring graft causes significantly less anterior knee pain and less incidence of kneeling pain $[5,11,21,28]$. Interestingly, patients tend to make a faster return to full hamstring tendon functionality following hamstring harvest than after patellar graft $[2,4,7,15,21]$. However, hamstring harvesting is complicated by the occurrence of numerous accessory bands originating from the GT and STT $[18,25]$.

Olewnik et al. [18] propose a sexpartite classification of accessory bands originating from the ST, GT and STT. The most common organisation was Type 1-1-1, i.e. monotendinous ST, GT and STT, which was observed in $52.9 \%$ of cases [18]. The second most frequent occurrence was Type 1-1-2, characterised by a monotendinous ST and GT, and one accessory band from the STT (31.4\%) [18]. The third most frequent occurrence was Type 1-1-3, which was characterised by monotendinous ST and GT and two accessory bands from the STT (8.8\%). Three more types were significantly less common: Type 1-2-3 (monotendinous ST, one accessory band from GT and two accessory bands from the STT; found in 1\%), Type 2-1-2 (one accessory band from ST, a single band from GT and one accessory band from STT; found in 2\%) and Type 2-2-3 (each muscle had one accessory band, one accessory band from the ST and GT and two accessory bands from the STT; found in 3.9\%) [18].

Based on Olewnik et al. [18] above, the tendon described in the present manuscript would be classified as Type 1-1-2, as it presents an accessory band originating from the STT. However, the accessory band then splits into two bands, where one inserts to the tibia and the other to the fascia of the soleus muscle. Although the classification could be modified to account for this form by creating a new Type 1-1-2 subtype, it would be better to add a separate rare cases group that defines the typical insertion of additional bands [18]. Additional bands originating from the GT attach to the fascia of the gastrocnemius muscle, while those originating from the STT join to the fascia of the gastrocnemius muscle, as well as to the deep fascia [18]. The case for an additional rare cases category is supported by the findings of Ashaolu et al. [1], who report an isolated example of a PA consisting of an ST, GT and STT, together with an accessory band of the STT, semi-membranous tendon and contributions from the tibial collateral ligament. An interesting case report by Rizvi et al. [25] also identified an unusual additional attachment of the STT and GT to the deep fascia. We believe that in addition to the case described above, both unusual types should also be categorised into "rare cases".

It is important to know the distance between PA insertion and the presence of additional bands during tendon procurement $[3,18,27]$. This distance has been found to be more than $10 \mathrm{~cm}$ in one study [3], between $6-8 \mathrm{~cm}$ and $8-12 \mathrm{~cm}$ from insertion by Tuncay et al. [27], and $6.35 \mathrm{~cm}$ from the main PA insertion $(6.35 \mathrm{~cm}$ mean length for the STT and $11.88 \mathrm{~cm}$ for the GT) by Olewnik et al. [18]. In the present case report, the accessory band was localised $80.79 \mathrm{~mm}$ from the main PA insertion: well within the $10 \mathrm{~cm}$ 'safe distance' given above.

The type of insertion also plays an important role when planning hamstring grafts. Fan-shaped and band-shaped types have previously been described [18]; however, the present case presents a band-shaped STT insertion and a fan-shaped GT insertion. In cases where the insertion is fan shaped, it might be harder to recognise the correct tendon and to expose the point where the tendon stripper can be placed [18].

\section{CONCLUSIONS}

The present case report describes a rare insertion of the accessory band of the STT, and determines the distance between the PA insertion and the additional band. This particular type of insertion could complicate the hamstring graft procedure. An awareness of the arrangement of the accessory bands of the semitendinosus and their insertion type (e.g. fascia of the soleus muscle) is important for the success of the harvesting procedure. The current classification should be extended to include such "rare cases" to improve future hamstring graft procedures.

\section{Ethical approval and consent to participate}

The cadaver belongs to the Department of Normal and Clinical Anatomy of the Medical University of Lodz. 


\section{REFERENCES}

1. Ashaolu JO, Osinuga S, Ukwenya VO, et al. Pes Anserinus structural framework and constituting tendons are grossly aberrant in nigerian population. Anat Res Int. 2015; 2015: 483186, doi: 10.1155/2015/483186, indexed in Pubmed: 26246910.

2. Bernicker JP, Haddad JL, Lintner DM, et al. Patellar tendon defect during the first year after anterior cruciate ligament reconstruction: appearance on serial magnetic resonance imaging. Arthroscopy. 1998; 14(8): 804-809, doi: 10.1016/s0749-8063(98)70014-3, indexed in Pubmed: 9848589.

3. Candal-Couto JJ, Deehan DJ. The accessory bands of Gracilis and Semitendinosus: an anatomical study. Knee. 2003; 10(4): 325-328, doi: 10.1016/s0968-0160(02)00154-0, indexed in Pubmed: 14629934.

4. Ferretti A, Conteduca F, Morelli F, et al. Regeneration of the semitendinosus tendon harvested for anterior cruciate ligament reconstruction. Evaluation using ultrasonography. Am J Sports Med. 2000; 28(4): 556-561, doi: 10.1177/03635465000280041901, indexed in Pubmed: 10921650.

5. Freedman KB, D'Amato MJ, Nedeff DD, et al. Arthroscopic anterior cruciate ligament reconstruction: a metaanalysis comparing patellar tendon and hamstring tendon autografts. Am J Sports Med. 2003; 31(1): 2-11, doi: 10.1177/03635465030310011501, indexed in Pubmed: 12531750.

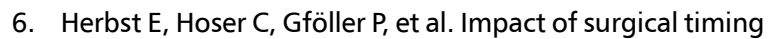
on the outcome of anterior cruciate ligament reconstruction. Knee Surg Sports Traumatol Arthrosc. 2017; 25(2): 569-577, doi: 10.1007/s00167-016-4291-y, indexed in Pubmed: 27549214.

7. Kartus J, Movin T, Karlsson J. Donor-site morbidity and anterior knee problems after anterior cruciate ligament reconstruction using autografts. Arthroscopy. 2001; 17(9): 971-980, doi: 10.1053/jars.2001.28979, indexed in Pubmed: 11694930.

8. Mahapatra P, Horriat S, Anand BS. Anterior cruciate ligament repair - past, present and future. J Exp Orthop. 2018; 5(1): 20, doi: 10.1186/s40634-018-0136-6, indexed in Pubmed: 29904897.

9. Mascarenhas R, MacDonald PB. Anterior cruciate ligament reconstruction: a look at prosthetics - past, present and possible future. Mcgill J Med. 2008; 11: 29-37.

10. Mochizuki T, Akita K, Muneta T, et al. Pes anserinus: layered supportive structure on the medial side of the knee. Clin Anat. 2004; 17(1): 50-54, doi: 10.1002/ca.10142, indexed in Pubmed: 14695588.

11. Mohtadi NG, Chan DS, Dainty KN, et al. Patellar tendon versus hamstring tendon autograft for anterior cruciate ligament rupture in adults. Cochrane Database Syst Rev. 2011; 9: CD005960, doi: 10.1002/14651858.CD005960. pub2, indexed in Pubmed: 21901700.

12. Moore KL, Dalley AF. Clinical Oriented Anatomy, 4th ed. Lippincott Williams \& Wilkins, Philadeplphia 1999.

13. Nau T, Lavoie $P$, Duval N. A new generation of artificial ligaments in reconstruction of the anterior cruciate ligament. Two-year follow-up of a randomised trial. J Bone Joint Surg Br. 2002; 84(3): 356-360, doi: 10.1302/0301-620x.84b3.12400, indexed in Pubmed: 12002492.

14. Nguene-Nyemb AG, Huten D, Ropars M. Chronic patellar tendon rupture reconstruction with a semitendinosus autograft. Orthop Traumatol Surg Res. 2011; 97(4): 447-450, doi: 10.1016/j.otsr.2011.01.015, indexed in Pubmed: 21596640.
15. Nixon RG, SeGall GK, Sax SL, et al. econstitution of the patellar tendon donor site after graft harvest. Clin Orthop Relat Res. 1995: 162-171.

16. Olewnik $t$, Gonera B, Kurtys K, et al. The anterolateral ligament of the knee: a proposed classification system. Clin Anat. 2018; 31(7): 966-973, doi: 10.1002/ca.23267, indexed in Pubmed: 30144325.

17. Olewnik $\measuredangle$, Gonera B, Kurtys K, et al. A proposal for a new classification of the fibular (lateral) collateral ligament based on morphological variations. Ann Anat. 2019; 222: 1-11, doi: 10.1016/j.aanat.2018.10.009, indexed in Pubmed: 30408521.

18. Olewnik $t$, Gonera B, Podgórski M, et al. A proposal for a new classification of pes anserinus morphology. Knee Surg Sports Traumatol Arthrosc. 2019; 27(9): 2984-2993, doi: 10.1007/s00167-018-5318-3, indexed in Pubmed: 30535546.

19. Olewnik $t$, Podgórski $M$, Polguj $M$, et al. The plantaris muscle - rare relations to the neurovascular bundle in the popliteal fossa. Folia Morphol. 2018; 77(4): 785-788, doi: 10.5603/FM.a2018.0039, indexed in Pubmed: 29651792.

20. Olewnik $\measuredangle$, Wysiadecki G, Podgórski M, et al. The plantaris muscle tendon and its relationship with the achilles tendinopathy. Biomed Res Int. 2018; 2018: 9623579, doi: 10.1155/2018/9623579, indexed in Pubmed: 29955614.

21. Paschos NK, Howell SM. Anterior cruciate ligament reconstruction: principles of treatment. EFORT Open Rev. 2016; 1(11): 398-408, doi: 10.1302/2058-5241.1.160032, indexed in Pubmed: 28461919.

22. Patel S, Trehan RK, Railton GT. Successful ACL reconstruction with a variant of the pes anserinus. J Orthop Traumatol. 2009; 10(4): 203-205, doi: 10.1007/s10195009-0075-1, indexed in Pubmed: 19921482.

23. Reina N, Abbo O, Gomez-Brouchet A, et al. Anatomy of the bands of the hamstring tendon: how can we improve harvest quality? Knee. 2013; 20(2): 90-95, doi: 10.1016/j. knee.2012.06.003, indexed in Pubmed: 22766187.

24. Risberg MA, Moksnes H, Storevold A, et al. Rehabilitation after anterior cruciate ligament injury influences joint loading during walking but not hopping. $\mathrm{Br} J$ Sports Med. 2009; 43(6): 423-428, doi: 10.1136/bjsm.2008.056846, indexed in Pubmed: 19273473.

25. Rizvi A, Iwanaga J, Oskouian RJ, et al. Additional attachment of the semitendinosus and gracilis muscles to the crural fascia: a review and case illustration. Cureus. 2018; 10(8): e3116, doi: 10.7759/cureus.3116, indexed in Pubmed: 30338191.

26. Takazawa $Y$, Ikeda $H$, Ishijima $M$, et al. Reconstruction of a ruptured patellar tendon using ipsilateral semitendinosus and gracilis tendons with preserved distal insertions: two case reports. BMC Res Notes. 2013; 6: 361, doi: 10.1186/1756-0500-6-361, indexed in Pubmed: 24010848

27. Tuncay I, Kucuker H, Uzun I, et al. The fascial band from semitendinosus to gastrocnemius: the critical point of hamstring harvesting: an anatomical study of 23 cadavers. Acta Orthop. 2007; 78(3): 361-363, doi: 10.1080/17453670710013933, indexed in Pubmed: 17611850

28. Xie X, Liu X, Chen Z, et al. A meta-analysis of bone-patellar tendon-bone autograft versus four-strand hamstring tendon autograft for anterior cruciate ligament reconstruction. Knee. 2015; 22(2): 100-110, doi: 10.1016/j. knee.2014.11.014, indexed in Pubmed: 25547048.

29. Yasin MN, Charalambous CP, Mills SP, et al. Accessory bands of the hamstring tendons: A clinical anatomical study. Clin Anat. 2010; 23(7): 862-865, doi: 10.1002/ ca. 21020, indexed in Pubmed: 20607820. 